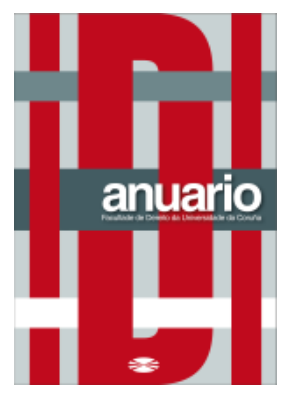

Anuario da Facultade de Dereito da Universidade da Coruña

Vol. 24 (2020), pp. 241-245

ISSNe: 2530-6324 || ISSN: 1138-039X

DOI: https://doi.org/10.17979/afdudc.2020.24.0.7499

\title{
KAHOOT!: UNA HERRAMIENTA INFORMÁTICA PARA LA MEJORA DE LA ENSEÑANZA Y EL APRENDIZAJE DEL DERECHO PROCESAL ${ }^{1}$
KAHHOT! A COMPUTER TOOL FOR THE IMPROVEMENT OF THE TEACHING AND LEARNING OF PROCEDURAL LAW

\author{
RUBÉN LÓPEZ PICÓ \\ Profesor Sustituto Interino de Derecho Procesal \\ Universidad de Granada \\ https://orcid.org/0000-0002-3243-5792
}

Recibido: 22/05/2020

Aceptado: 08/06/2020

Resumen: A través de estas líneas, tratamos de poner de manifiesto como la incorporación de las Tecnologías de la Información y de la Comunicación -TIC-, en general, y de la plataforma Kahoot!, en particular, a los nuevos y modernos métodos universitarios puede resultar de gran utilidad para la mejora de la enseñanza y el aprendizaje del Derecho Procesal.

Palabras claves: Innovación Docente, Plataforma Kahoot!, Derecho Procesal.

Abstract: Through these lines, we try to show how the incorporation of the Information and Communication Technologies -ICT-, in general, and of the Kahoot! platform, in particular, to the new and modern university methods can be very useful for the improvement of the teaching and learning of Procedural Law.

Keywords: Teaching Innovation, Kahoot! Platform, Procedural Law.

\footnotetext{
${ }^{1}$ El presente trabajo de Innovación Docente se enmarca dentro del Proyecto de Innovación y Mejora de la Calidad Docente "La corrección del discurso jurídico en un contexto de aula invertida" (PID 192020), financiado por el Instituto de Investigación e Innovación Educativa de la Universidad de las Islas BalearesGobierno de las Islas Baleares, cuyo IP es Felip Alba Cladera.
} 


\section{Sumario: I. INTRODUCCIÓN. II. PLATAFORMA KAHOOT! III. CONCLUSIONES. BIBLIOGRAFÍA.}

\section{INTRODUCCIÓN}

El nuevo modelo educativo European Credit Transfer System -ECTS-, impuesto por el Espacio Europeo de Educación Superior -EEES- y centrado en el aprendizaje autónomo de los estudiantes bajo la supervisión del profesorado universitario responsable de las diferentes asignaturas, lleva consigo aparejado la necesidad de repensar las actividades de enseñanza y aprendizaje universitario; sobre todo teniendo presente la enorme importancia atribuida al uso de las Tecnologías de la Información y de la Comunicación -TIC- como herramientas enormemente útiles para la elaboración, adquisición y transmisión del conocimiento dentro de este novedoso modelo educativo universitario ${ }^{2}$.

Para poder hacer frente a este nuevo reto, es necesario que el profesorado universitario experimente un cambio en lo relativo al papel o rol que desempeña, al menos en lo que a su participación en el proceso de enseñanza se refiere. Debiendo apostar, en consecuencia, por el desarrollo de modernos e innovadores métodos pedagógicos capaces de integrar el uso de las TIC en el sistema de enseñanza y aprendizaje universitario.

Conscientes de esta realidad, defendemos la necesidad de incorporar el uso de las TIC a nuestra labor como docentes universitarios y, por consiguiente, desarrollar nuevos y modernos métodos de enseñanza universitaria que, en nuestro caso concreto, nos permitan incorporar o integrar las TIC a la enseñanza universitaria del Derecho Procesal.

Junto a las nuevas exigencias educativas impuestas por el EEES, nuestra preocupación por que los estudiantes universitarios, además de las propias de la materia impartida -Derecho Procesal, en nuestro caso-, adquieran las denominadas competencias genéricas -recordemos: trabajo cooperativo, capacidades de comunicación, experiencia profesional...- que, en un futuro próximo, les serán requeridas en el momento de acceder al mercado laboral, nos lleva a tenerlas presente durante la confección de los nuevos y modernos métodos de enseñanza y aprendizaje universitario antes referidos. Todo ello sin llegar a olvidar que las lecciones magistrales impartidas por el profesorado universitario experto en la materia constituyen la base o el fundamento de todo el proceso de enseñanza y aprendizaje universitario.

${ }^{2}$ LÓPEZ PICÓ, R., "El empleo de las nuevas tecnologías en la enseñanza del Derecho Procesal”, en SÁNCHEZ-ARCHIDONA HIDALGO, G., GARCÍA PASTOR, A.M., y VALENCIA SÁIZ, A. (Coord.), Desafíos actuales del Derecho: aportaciones presentadas al II Congreso Nacional de Jóvenes Investigadores en Ciencias Jurídicas, Ed. EUMED, Málaga, 2020, p. 1357. En este mismo sentido, GARRIDO CARRILLO, F.J. y ARTACHO MARTÍN-LAGOS, M., "Nuevas tecnologías y adquisición de competencias en la enseñanza del Derecho", en GARRIDO CARRILLO, F.J. (Dir.), Intercambios y buenas prácticas en la enseñanza del Derecho. Nuevos Métodos Docentes, Ed. Comares, 2012, Granada, pp. 163-170. 


\section{PLATAFORMA KAHOOT!}

La plataforma Kahoot! constituye una herramienta informática que -estando ubicada dentro de los diferentes programas SRS (Student Response Systems)- nos permite obtener de forma instantánea y ordenada toda la información emitida por los estudiantes en forma de contestación a las preguntas que previamente les han sido formuladas con relación a algún aspecto concreto de la materia que integra o compone el programa de la asignatura. Toda esa información se puede guardar y exportar de un modo muy sencillo a través de la creación del oportuno Excel -aspecto esencial para la evaluación individual de los estudiantes y la estadística de aprendizaje activo- resultado de su facilidad de creación y aplicación ${ }^{3}$.

De forma previa al inicio de la lección magistral en la que pretendemos hacer uso de esta herramienta informática, el profesorado universitario responsable de la asignatura deberá crear la oportuna sesión dentro de la plataforma Kahoot! -web https://Kahoot.com/e introducir -una vez registrado (gratuitamente y mediante el empleo de una dirección electrónica)- las diferentes preguntas que se desean formular a los estudiantes. Además de introducir las preguntas en la presentación interactiva, el profesorado también deberá indicar cuál es la respuesta correcta dada a cada una de las preguntas realizadas, para que, tras recibir las contestaciones de los estudiantes, el programa informático pueda proceder a emitir el correspondiente informe. De entre sus diferentes modalidades de pruebas, se aconseja el empleo de la opción quiz al ser ésta la única modalidad que permite efectuar preguntas con múltiples respuestas posibles -MCQ-.

El objetivo esencial que se pretende alcanzar, a través del uso de esta herramienta informática e interactiva, es que cada estudiante a través del uso de la misma pueda dar respuesta a las cuestiones que se le plantean aplicando los conceptos básicos que previamente han sido explicados durante el desarrollo de las lecciones magistrales impartidas por el profesorado universitario responsable de la asignatura. Tras responder a las preguntas planteadas, el sistema informático crea el histograma de respuestas, que, tras ser proyectado en la clase, sirve para que los estudiantes puedan conocer los resultados alcanzados por ellos mismos y por cada uno de sus compañeros; permitiéndoles, en consecuencia, conocer el nivel de estudios y el grado de asimilación alcanzado hasta el momento ${ }^{4}$.

Entre sus diferentes opciones de uso -individual (classic) o colectivo (team mode), apostamos por la modalidad "classic". Así, creada la sesión por el profesorado universitario responsable de la asignatura, el propio programa informático genera un código pin que los estudiantes participantes en esta experiencia de innovación docente deben

\footnotetext{
${ }^{3}$ SOLANES GIRALT, M.M., "Gamificación con los juegos pasapalabra, crucigrama y Kahoot para la evaluación del aprendizaje del derecho en el aula invertida”, en DELGADO GARCÍA, A.M. y DE HEREDIA RUIZ, I.B. (Coord.), La Docencia del Derecho en la Sociedad Digital, Ed. Huygens, Barcelona, 2019 , p. 201.

${ }^{4}$ HUALDE LÓPEZ, I. y SÁNCHEZ POS, Ma . V., "La utilización de los Clickers como herramienta de apoyo a la docencia del Derecho Procesal”, en PICÓ I JUNOY, J. (Dir.), El aprendizaje del Derecho Procesal: nuevos retos en la enseñanza universitaria, Ed. Bosch, Barcelona, 2011, pp. 437 y 438.
} 
introducir en sus dispositivos informáticos -smartphone, tablets u ordenadores- ${ }^{5}$. Una vez hecho esto, deberán identificarse utilizando su nombre y apellidos.

Al igual que cualquier otra herramienta informática implementada en los nuevos y modernos métodos universitarios diseñados para la mejora de la enseñanza y aprendizaje del Derecho Procesal, el uso de la plataforma Kahoot! como herramienta informática de apoyo al desarrollo de las lecciones magistrales impartidas por el profesorado universitario experto en la materia, únicamente nos permitirá obtener los múltiples e importantes beneficios pretendidos a través de su empleo, cuando precisamente éste último tenga lugar de forma correcta. De ahí, la enorme importancia de que el profesorado universitario que desea hacer uso de la plataforma Kahoot! durante el desarrollo de sus propias lecciones magistrales conozca todo su potencial y correcto funcionamiento.

Es por ello que el empleo de esta herramienta informática, como medio de innovación docente para la enseñanza y aprendizaje del Derecho Procesal, obliga al profesorado universitario responsable de la asignatura en la que se pretende hacer uso de la misma a adquirir nuevas competencias no sólo en lo relativo a los aspectos técnicos relacionados con su empleo sino también a la planificación de las clases, la redacción de las preguntas que desea formular a los estudiantes y la moderación del debate que con carácter posterior se pudiese originar. Para la adquisición de estas nuevas competencias, el profesorado universitario puede optar por recibir la oportuna instrucción de manos de otros profesores universitarios ya experimentados en el uso de la plataforma Kahoot! o del personal laboral de la Universidad dedicado a la formación del profesorado universitario ${ }^{6}$.

\section{CONCLUSIONES}

La implementación de las herramientas informáticas, derivadas del desarrollo de las TIC, en los nuevos y modernos métodos universitarios diseñados para la mejora de la enseñanza y aprendizaje del Derecho Procesal constituye la más que evidente materialización del nuevo modelo educativo European Credit Transfer System -ECTSimpuesto por el Espacio Europeo de Educación Superior -EEES-.

La posibilidad de poder combinar las tradicionales lecciones magistrales impartidas por el profesorado universitario experto en la materia con el uso de las nuevas y modernas tecnologías -plataforma Kahoot!, en este supuesto concreto-, permiten al estudiantado universitario adquirir los conocimientos de Derecho Procesal necesarios para conseguir superar la asignatura $\mathrm{y}$, contemporáneamente, la adquisición de otras competencias que les serán exigibles en el momento de acceder al mercado laboral.

${ }^{5}$ A diferencia de otras herramientas informáticas implementadas también en los nuevos y modernos métodos diseñados para el aprendizaje y la enseñanza universitaria del Derecho Procesal, la plataforma Kahoot! no requiere que el aula en la que habitualmente se imparten las lecciones magistrales esté dotada de los últimos y más modernos medios materiales e informáticos, pues su empleo es perfectamente posible a través de smartphone, tablets y ordenadores.

6 LÓPEZ PICÓ, R., “El uso de Clickers en la enseñanza del Derecho Procesal”, en ALONSO SALGADO, C. y OTERO CRESPO, M. (Dir.), Investigación y Docencia en Derecho: Nuevas perspectivas, Ed. Colex, A Coruña, 2019, pp. 104 y 105. 
Independientemente de la herramienta informática que el profesorado universitario decida utilizar como instrumento de apoyo para el desarrollo de sus lecciones magistrales, éstas -herramientas informáticas- deben ser empleadas de forma eficaz y eficiente a fin de evitar que la monotonía en su empleo origine la desconexión de los estudiantes durante el desarrollo de las lecciones magistrales. Y es que pese a las enormes virtudes que el uso de estas nuevas y modernas herramientas informáticas presenta, su principal peligro radica precisamente en su capacidad para distraer al estudiantado universitario. Recordemos que el protagonista absoluto durante la impartición de las lecciones magistrales es y debe ser el profesorado universitario experto en la materia y responsable de la asignatura, y no la herramienta o las herramientas informáticas utilizadas como instrumentos de apoyo.

\section{BIBLIOGRAFÍA}

GARRIDO CARRILLO, F.J. y ARTACHO MARTÍN-LAGOS, M., “Nuevas tecnologías y adquisición de competencias en la enseñanza del Derecho”, en GARRIDO CARRILLO, F.J. (Dir.), Intercambios y buenas prácticas en la enseñanza del Derecho. Nuevos Métodos Docentes. Ed. Comares, 2012, Granada.

HUALDE LÓPEZ, I. y SÁNCHEZ POS, Ma . V., "La utilización de los Clickers como herramienta de apoyo a la docencia del Derecho Procesal”, en PICÓ I JUNOY, J. (Dir.), El aprendizaje del Derecho Procesal: nuevos retos en la enseñanza universitaria. Ed. Bosch, Barcelona, 2011.

LÓPEZ PICÓ, R., "El uso de Clickers en la enseñanza del Derecho Procesal”, en ALONSO SALGADO, C. y OTERO CRESPO, M. (Dir.), Investigación y Docencia en Derecho: Nuevas perspectivas, Ed. Colex, A Coruña, 2019.

LÓPEZ PICÓ, R., “El empleo de las nuevas tecnologías en la enseñanza del Derecho Procesal”, en SÁNCHEZ-ARCHIDONA HIDALGO, G., GARCÍA PASTOR, A.M., y VALENCIA SÁIZ, A. (Coord.), Desafíos actuales del Derecho: aportaciones presentadas al II Congreso Nacional de Jóvenes Investigadores en Ciencias Jurídicas. Ed. EUMED, Málaga, 2020.

SOLANES GIRALT, M.M., "Gamificación con los juegos pasapalabra, crucigrama y Kahoot para la evaluación del aprendizaje del derecho en el aula invertida”, en DELGADO GARCÍA, A.M. y DE HEREDIA RUIZ, I.B. (Coord.), La Docencia del Derecho en la Sociedad Digital. Ed. Huygens, Barcelona, 2019. 